\title{
Objective Accommodation Amplitude Measurements Using a New Autorefractometer Device
}

\author{
(D) Kemal Ozulken, ${ }^{1}$ (D) Hasan Kiziltoprak ${ }^{2}$ \\ 1Department of Ophthalmology, TOBB ETU Medical Faculty Hospital, Ankara, Turkey \\ 2Department of Ophthalmology, Bingol Women's Health and Children's Hospital, Bingol, Turkey
}

\begin{abstract}
Objectives: To measure the value of objective accommodation amplitude (AA) in patients using the new autorefractometer device and to evaluate the effects of age, refraction errors, pupil diameter on objective AA measurements.

Methods: Three hundred subjects who were divided into five groups according to age were enrolled in this study. AA and pupil diameter were measured three times from both eyes using Tonoref III (NIDEK Co., Ltd.).

Results: The mean AA was I.6325 \pm 0.06 I Diopter (D) (0.13-9.II D). The mean AA values were statistically significantly different between the groups $(p=0.000)$. It was not observed gender effect on $A A(p=0.115)$. Although there was no significant difference between emmetropic and myopic groups, there were significant differences between emmetropic and hyperopic groups and also between myopic and hyperopic groups concerning $A A(p=0.000$, for both). A statistically significant difference was found for the mean AA between the groups with and without refractive surgery $(p=0.028)$. Correlation analysis revealed that AA is increased as the mean pupil diameter increases $(p=0.000, r=0.202)$ and the mean pupil diameter decreases with increasing age $(p=0.000, r=-0.308)$. When the AA obtained from the patients in group I were compared with the AA values obtained by subjective accommodation tests, the AA values obtained by autorefractometer device were found to be correlated with push up, push down and minus lens tests $(r=0.577, r=0.682, r=0.427)$ AA values obtained by autorefractometer device were found to be statistically significantly lower than other subjective tests $(p<0.001)$.

Conclusion: The Tonoref III device seems to provide objective AA measurements in presbyopic or non-presbyopic individuals. Age, refractive error, pupil diameter and history of refractive surgery were effective on AA values, while the gender was not effective.
\end{abstract}

Keywords: Amplitude of accommodation, autorefractometer, objective accommodation test, pupil diameter, presbyopia.

\section{Introduction}

Accommodation is an optical change in the power of the eye when looking at a close distance from a far distance (I). The accommodation decreases with advancing age, which results in loss of vision in the near-term objects called presbyopia (2, 3). In humans, the decline in accommodation amplitude (AA) results in a complete loss of near vision starting in the youth (4). Although changes in most of the anatomical structures, such as modified ciliary body configuration and loss of capsule flexibility, are associated with age, the most important change is the increase in the stiffness of the intraocular lens $(I, 4)$.

For the surgical restoration of active and dynamic accommodation in the presbyopic eyes, an attempt is made to develop designed intraocular lenses (IOLs) or scleral enlargement procedures to create a real change in the optical power of the intraocular lens during accommodation (5-7).

Address for correspondence: Hasan Kiziltoprak, MD. Bingol Kadin Sagligi ve Cocuk Hastanesi, Oftalmoloji Klinigi, Bingol, Turkey

Phone: +90 4263126261 E-mail: hsnkzltprk21@gmail.com

Submitted Date: July 19, 2019 Accepted Date: November 26, 2019 Available Online Date: December 25, 2019

${ }^{\circ}$ Copyright 2019 by Beyoglu Eye Training and Research Hospital - Available online at www.beyoglueye.com OPEN ACCESS This work is licensed under a Creative Commons Attribution-NonCommercial 4.0 International License. 
Despite subjective clinical evaluations showed high patient satisfaction and good visual performance, accommodation performance measured objectively with these procedures was disappointing. Although patient satisfaction is important, efforts to improve the performance of accommodation restoration procedures require objective measurements to understand their mechanisms of action.

The subjective accommodation tests, such as the clinical push-up test, minus lens method used routinely, are not suitable for the precise demonstration of an eye-catching optical change in the power of the eye (8-II). Subjective tests do not reveal the change in passive depth of field due to small pupil diameter and ocular aberrations and active accommodation in the eye (9-12). In addition, subjective tests significantly overestimate the actual accommodation change when accommodation is available $(9,12)$. Thus, several methods, such as dynamic retinoscopy (DR) and autorefractometer, are available for objective AA measurements (12-15). The Tonoref III (NIDEK Co., Ltd, Gamagori, Japan) is a recently developed an autorefractometer device that has multiple functions, such as auto-refractor, keratometer, non-contact tonometer and non-contact optical pachymeter (16). The Tonoref III can also measure AA and can give us a pupil diameter at the same time (16). The autorefractor function measures AA by focusing on a target approaching from a far distance.

In this study, we aimed to measure the objective AA in presbyopic and non-presbyopic participants using the Tonoref III device and to investigate the effects of age, gender, refraction errors, pupil diameter and history of refractive surgery on AA.

\section{Methods}

This study was conducted at a tertiary university hospital. Three hundred subjects who admitted to our clinic for a routine ophthalmic examination were included in this study. The study adhered to the tenets of the Declaration of Helsinki and the study protocol was approved by the institutional review board of the ethical committee.

Exclusion criteria were as follows: Best-corrected visual acuity (BCVA) lower than 20/25 on Snellen equivalent; spherical refractive error higher than $6.00 \mathrm{D}$ of hyperopia or myopia; cylindrical refractive error higher than $2.00 \mathrm{D}$; any ocular pathology including cataract, strabismus, amblyopia, glaucoma, uveitis; any systemic disorder including diabetes mellitus, childhood diphtheria. In addition, any subjects reporting a history of significant ocular or head injury, history of retinal photocoagulation or ocular surgery except refractive surgery or history of prolonged use of topical cycloplegics, phenothiazines, tricyclic antidepressants and antivertigo drugs that may affect accommodation were also excluded from this study.

All patients underwent a comprehensive ophthalmologic examination, including BCVA, assessment of the anterior and posterior segments of the eye using a slit-lamp and direct ophthalmoscopy through an undilated pupil, respectively. An emmetropia was defined as one in which the spherical correction is less than or equal to $\pm 0.50 \mathrm{D}$. Hyperopia and myopia were defined as any eye with a spherical correction of more than or equal to $+0.50 \mathrm{D}$ and $-0.50 \mathrm{D}$, respectively.

The participants were divided into five groups according to their age: The ages of 25-29 years were identified as group I, 30-34 years as group 2, 35-39 years as group 3, 40-49 years as group 4, 50 years and over as group 5 . The BCVA was obtained after undilated autorefractometer measurements. A duochrome test was carried out for all patients to prevent over or under correction for distance.

For objective measurement using Tonoref III, the subjects were seated at the instrument with his or her head stabilized in the instrument chin rest and forehead strap. To maintain a stable pupil, room illumination was dimmed and was measured to be 0.1 lux at the headrest of the instruments where the subject sat. All measurements were performed at the same environmental conditions and the same time frame of the day (10:00-12:00 AM). Three consecutive measurements were made and means of these three values were recorded as AA. To stimulate accommodation, a chart as the fixation target ( 0.63 Snellen chart) was moved to the initial position based on the measurement result of objective refractive value, and astigmatism of patients' eyes were corrected automatically by Tonoref III. Then, measurement started when performing the alignment. The chart moves to minus side to apply accommodative stimulus (from +20 D TO -20D), and Tonoref III measures refractive index continuously while applying accommodative stimulus. The device measures AA between 0-10 D (0.01,0.12,0.25 increments), pupil diameter between 3 to $8.5 \mathrm{~mm}$ ( $1 \mathrm{~mm}$ increments). Refractive value of accommodation is only use central area. Measurement finishes under the specified condition: there is no change in accommodation power for more than six seconds or when measurement time reaches 30 seconds. Finally, the result was shown on a graph indicating accommodation power and change in refractive value. AA measured as difference between maximum and minimum refractive value. The pupil diameter was also detected with AA measurements.

To determine the correlation between objective AA values obtained with new Autorefractometer device and other subjective accommodation tests, non-presbyopic patients in group I were subjected to push-up, push-down and minus lens subjective accommodation tests.

For the push-up method, the subjects initially viewed the target at a distance of approximately $40 \mathrm{~cm}$. Then, the target 
was moved slowly toward him/her along the ruler. The subjects were instructed to keep the target as clear as possible and to report when it first became blurred. The endpoint was the first slight sustained blur, which was considered to be the point when the target could not be cleared after two or three seconds of viewing. The distance from the target to the spectacle plane was measured with a millimeter ruler and converted to diopters.

In the push-down method, the accommodative target was advanced toward the subject until a significant blur was produced, and then, the target was pushed away until the subject could just read the 20/20 row of letters. Again, the distance from the target to the spectacle plane was measured and converted to diopters.

In the minus lens procedure, a reduced Snellen chart was positioned at $40 \mathrm{~cm}$. The subjects were asked to fixate on the $20 / 20$ row of letters on the chart, while a minus power was added (in $0.25 \mathrm{D}$ steps) to the previously-determined subjective refraction until the letters became, and remained, blurred. The ML amplitude was taken as $2.50 \mathrm{D}$ (the dioptric equivalent of the working distance) plus the amount of minus lens power was added.

\section{Statistical Analysis}

Statistical evaluation of the data was performed using the Statistical Package for Social Sciences (SPSS) version 20.0. In the analysis of numerical data, conformity to normal distribution was examined using Kolmogorov Simonov and ShapiroWilk tests. Since all the data were not normally distributed, Mann-Whitney $U$ was used for paired group comparisons, and Kruskal Wallis $\mathrm{H}$ tests were used for multiple group comparisons. The correlation coefficient and significance were determined using the Spearman method. If the $p$-value was less than 0.05 , the tests were accepted as significant.

\section{Results}

This study included a total of 600 eyes of the 300 patients. Of the 300 patients, 179 (59.7\%) were female and 12I $(40.3 \%)$ were male. The mean age of the participants was $39.06 \pm 0.608$ years (25-65 years). From all participants, 284 $(47.3 \%)$ of the eyes were myopic, $124(20.7 \%)$ were hyperopic, and 192 (32\%) were emmetropic. Twenty-six eyes had refractive surgery. The age group, gender and refractive status of the patients are shown in Table $I$.

The mean AA of the eyes were $1.6325 \pm 0.061 \mathrm{D}(0.13$ 9.II D). The mean AA values were statistically significantly different between the groups ( $p=0.000$, for each) (Table 2$)$. The findings showed that there was a statistically significant difference between the age groups and mean AA in both genders $(p=0.000)$. As age increased, the accommodation value decreased. There was no statistically significant difference between the mean $A A$ and the gender $(p=0.1$ I5) (Figs. I, 2).
Table I. The age group, gender and refractive status of the patients Patients (n) $\%$

\begin{tabular}{lcc}
$\begin{array}{l}\text { Gender } \\
\text { Female }\end{array}$ & 179 & 59.7 \\
Male & 121 & 40.3 \\
Age group & & \\
$25-29$ & 63 & 21.0 \\
$30-34$ & 56 & 18.7 \\
$35-39$ & 55 & 18.3 \\
$40-49$ & 79 & 26.3 \\
50 and over & 47 & 15.7 \\
Total & 300 & 100.0 \\
& Eyes (n) & $\%$ \\
\hline Spherical group & & \\
Emetrope & 192 & 32.0 \\
Myopia & 284 & 47.3 \\
Hyperopia & 124 & 20.7 \\
LASIK group & & 95.7 \\
Negative & 574 & 4.3 \\
Positive & 26 & 100.0 \\
Total & 600 & \\
\hline & &
\end{tabular}

Table 2. The mean accommodation amplitude values in age groups

\begin{tabular}{lcc} 
Age group & $\begin{array}{c}\text { Mean accommodation amplitude } \\
\text { /Standard error/Min-Max }\end{array}$ & p \\
\hline $25-29$ & $3.1717 \pm 0.16488(0.37-9.11)$ & 0.000 \\
$30-34$ & $2.0419 \pm 0.11372(0.35-5.13)$ & \\
$35-39$ & $1.6935 \pm 0.11026(0.23-5.58)$ & \\
$40-49$ & $0.7678 \pm 0.03598(0.14-2.60)$ & \\
50 and over & $0.4632 \pm 0.02636(0.13-1.52)$ & \\
Total & $1.6325 \pm 0.061(0.13-9.11)$ & \\
\hline
\end{tabular}

In the analysis of refractive errors on AA, there was a statistically significant difference between the refractive groups for AA ( $p=0.000)$ (Table 3$)$. In detailed subgroupanalysis, there was no significant difference between the emmetropic group and myopia group, but there were significant differences between emmetropia and hyperopia also between myopia and hyperopia groups $(p=0.000)$.

The mean AA was compared between emmetropic 26 eyes of 13 patients who had undergone laser in situ keratomileusis (LASIK) for myopia and 26 eyes of 13 age and sex-matched patients who had not undergone LASIK randomly selected among emmetropic patients. A statistically significant difference was found for mean AA between the group with and without LASIK $(p=0.028)$ (Table 4). 


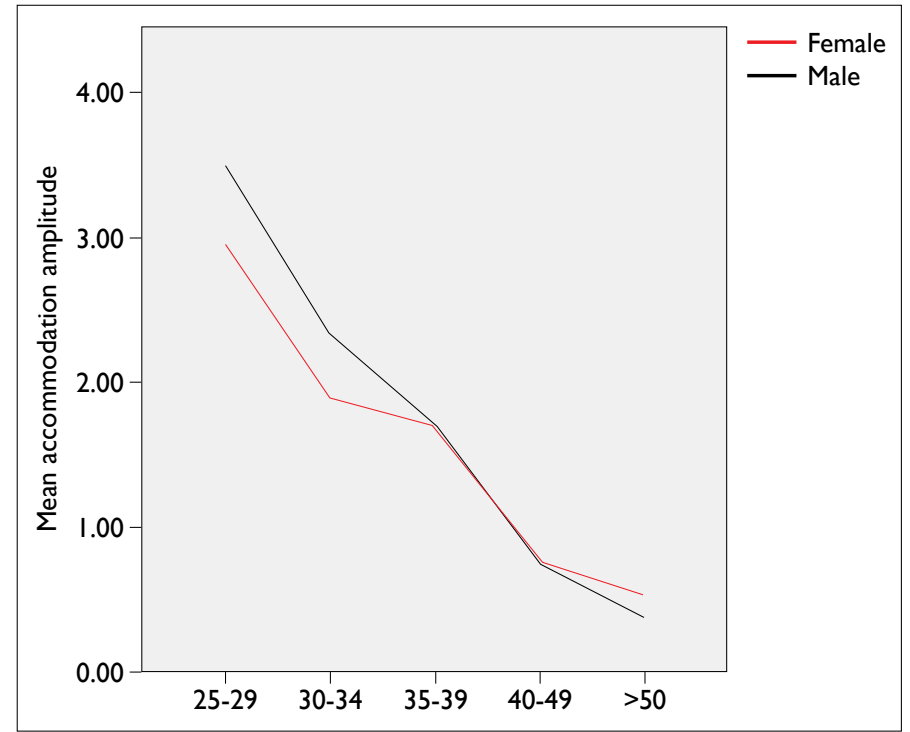

Figure I. The mean accommodation amplitude changes by age in different genders.

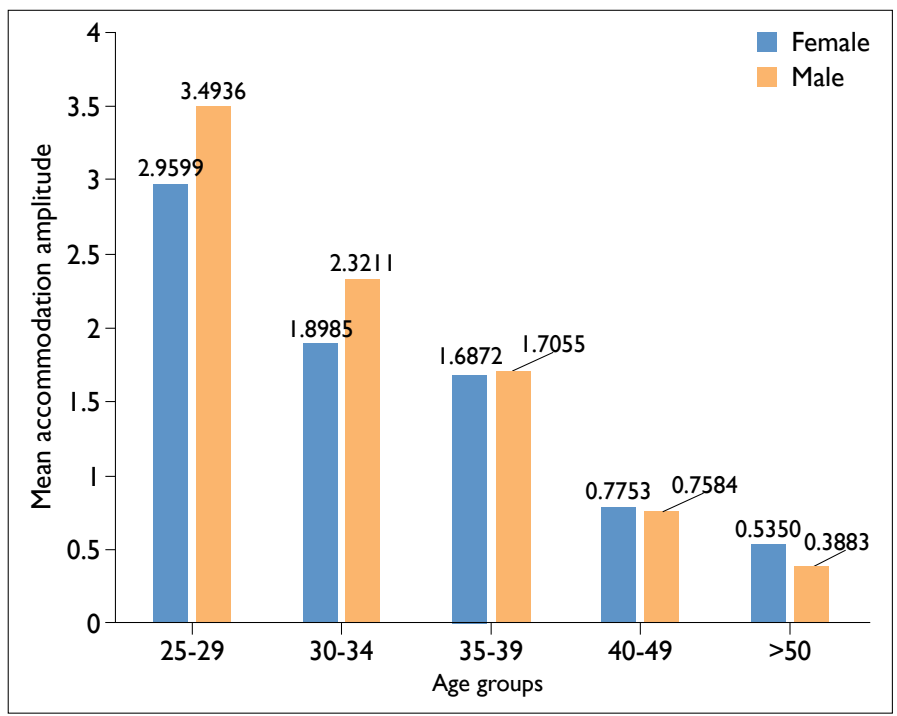

Figure 2. The mean accommodation amplitude values in different age groups and genders.

It was also observed that the $\mathrm{AA}$ increased as the mean pupil diameter increased $(p=0.000, r=0.202)$, and the mean pupil diameter decreases with increasing age $(p=0.000, r=$ 0.308) (Fig. 3).

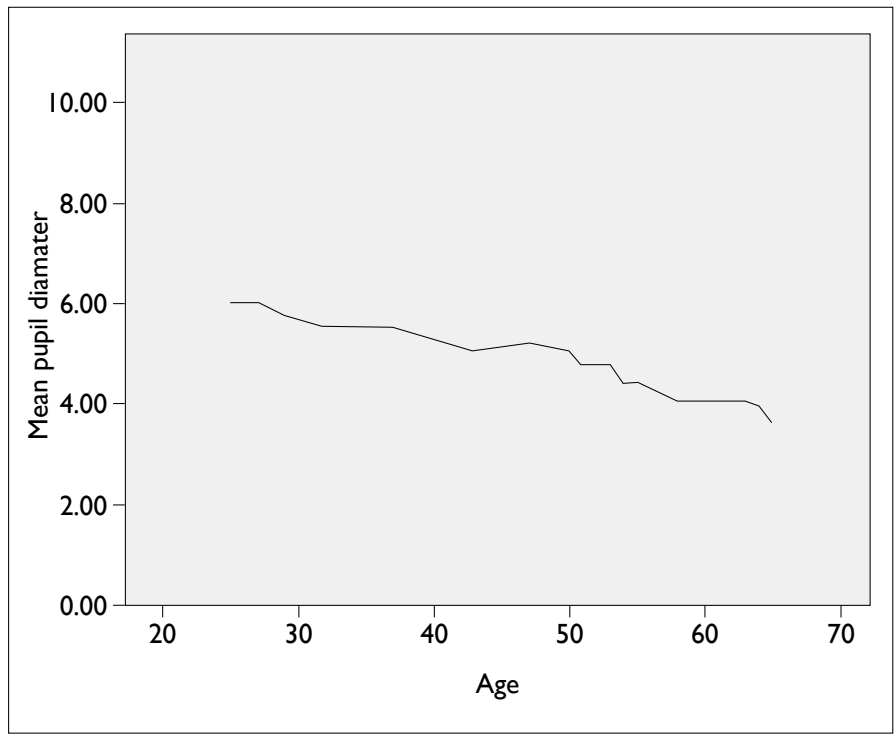

Figure 3. The mean pupil diameter changes by age.

In the non-presbyopic patients between the ages of 25-29 in Group I, AA values obtained by autorefractometer were compared with subjective accommodation tests. Mean AA values obtained by push up, push down and minus lens tests were as follows: $5.70 \pm 0.09,4.7 I \pm 0.08$, and $7.52 \pm 0.07$ and the AA values obtained by autorefractometer were statistically significantly lower than those of the other three subjective accommodation tests $(p<0.00 \mathrm{I})$ (Table 5). Moreover, in group I, it was investigated whether there was a correlation between $A A$ values obtained with autorefractometer device and $A A$ values obtained in other subjective tests. AA values obtained with autorefractometer device correlated with push up, push down and minus lens tests $(r=0.577, r=0.682$, $r=0.427$ ) (Fig. 4).

\section{Discussion}

In this study, we evaluated the newer autorefractometer device to determine the ability of the instrument to measure AA reliably. AA was measured with that device that was consistent with the normal distribution as it decreased with increasing age. We also evaluated factors affecting the measurements, such as age, gender, pupil diameter, refractive errors and history of LASIK surgery.

Table 3. The mean accommodation amplitude values in different refractive groups

\begin{tabular}{lccc} 
Refractive group & Number of eyes & Mean accommodation amplitude & p \\
\hline Myopia & 284 & 1.8496 & $0.000^{*}$ \\
Emmetropia & 192 & 1.7806 & \\
Hyperopia & 124 & 0.9057 & \\
Total & 600 & 1.6325 & \\
\hline
\end{tabular}


Table 4. The mean accommodation amplitude in LASIK and Non-LASIK groups

\begin{tabular}{lccc} 
LASIK & Number of eyes & $\begin{array}{c}\text { Mean accommodation amplitude } \\
\text { /Standard error/Min-Max }\end{array}$ & p \\
\hline Negative & 26 & $1.4835 \pm 0.24171(0.21-4.59)$ & 0.012 \\
Positive & 26 & $2.8027 \pm 0.37179(0.30-5.95)$ & \\
Total & 52 & $2.1431 \pm 0.23818(0.21-5.95)$ & \\
\hline
\end{tabular}

Table 5. The mean accommodation amplitude values in different accommodation tests

\section{Mean/Standard error/Min-Max}

\begin{tabular}{lc}
\hline Objective autorefractometer & $3.1717 \pm 0.16488(0.37-9.1 \mathrm{I})$ \\
Push up & $5.7008 \pm 0.0970(4.00-7.5 \mathrm{I})$ \\
Push down & $4.71363 \pm 0.08623(3.56-6.22)$ \\
Minus lens & $7.52540 \pm 0.0768 \mathrm{I}(6.00-8.45)$ \\
\hline
\end{tabular}

AA can be measured by different techniques as subjective and objective tests. Subjective methods, include the minus lens method, push down and push up test $(4,8)$. DR and automated refractometer are used for objective accommodation measurements (I, I4, I7). Several studies evaluate these techniques and it was found that subjective methods typically overestimate true AA. Hence, the subjective tests do not differentiate between passive depth of field due to small pupils and ocular aberrations and active accommodative power change in the eye $(4,12)$. The autorefractometer objectively calculate the difference between the distance refraction and near refraction and excludes subjective factors, such as depth of focus of the eye. Therefore, objective methods detect $A A$ more reliably than subjective methods ( $I, 4$, 17). In previous studies, although autorefractometers were used, they showed distant and near chart and measured refraction. In addition, the patients' refractive defects were corrected by contact lenses. In our study, the device corrects astigmatism and measures the refraction as the chart moves and gives the AA. This technique seems to make the measurements very easy and completely objective. In our study, when we compared our results with subjective tests in non-presbyopic patients, we found that AA values obtained with autorefractometer device were correlated with other subjective tests and were significantly lower. Our findings support that subjective accommodation tests overestimate AA values, similar to the literature.

To study AA changing by different ages, Leon et al. (14) evaluated 1410 subjects between five and 60 years of age using DR and showed a significant negative correlation between age and the AA. Similarly, a metanalysis reported that subjective AA decreases approximately linearly with age until about age 40 or 45 years at about $0.3 \mathrm{D}$ per year (I8). We also found a decrease in AA with increasing age. However, AA measured with Tonoref III was lower than that measured with DR until the age of 45 years. In presbyopic groups, both methods had similar AA measurements. Another study evaluated the AA in the prepresbiyopic group aged between 21 to 30 years by WR-5I00K autorefractor (Grand Seiko Co., Ltd.) also had higher levels of AA than our measurements (I2).

The $A A$ and its relationship with refractive errors have been studied in several studies. McBrien et al. (19) evaluated the AA in students aged between 18 and 22 years and found that the $A A$ was highest in late-onset myopia and least in hyperopia. Similarly, Leon et al. (14) found significant differences between the values of AA for emmetropia and hyperopia and between myopia and hyperopia by dy-

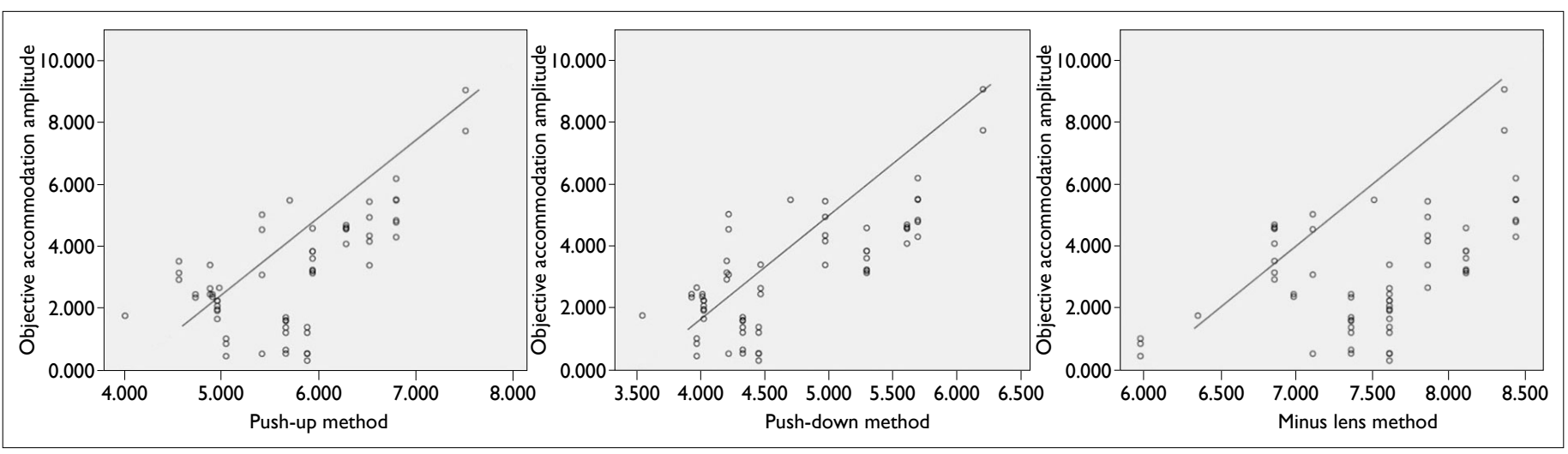

Figure 4. Correlation analysis of objective autorefractometer accommodation test with subjective methods. 
namic retinoscopy. However, no significant difference in AA between emmetropia and myopia was observed. Another study also revealed similar results as myopic patients had higher AA than emmetropic hypermetropic patients, but after the age of 44 years, the AA converges to similar values in the three refractive groups (20). On the other hand, Schaeffel et al. (2I) have shown that refractive errors do not affect the dynamics of natural accommodation. In our study group, a significant difference was found between hypermetropic patients and myopic or emmetropic patients. Myopic and emmetropic patients had higher AA than hypermetropic patients.

Accommodation generally accompanied by pupillary constriction (miosis) and binocular convergence. As known, pupil constriction has a positive effect on the size of the blurred image. Hence, accommodative miosis is important for good or acceptable retinal image quality in case of impaired accommodation. Miosis also increases the patient's subjective AA as the blurred image decreases. Lopez-Alcon et al. (I8) showed pupil diameter and its change during accommodation were not affect subjective AA significantly even though its effect on fourth-sixth-order spherical aberration strongly depends on the pupil size. On the other hand, a study conducted by Lara et al. (22) indicates that AA can be dependent on pupil size in two ways. Firstly, a changing pupil size will change spherical aberration and thus measured AA. Secondly, when the pupil size is increased by lowering the ambient light levels, subjects reliably accommodated less because of the multifocality of the eye with large pupils and the accompanying increased depth of field. Therefore, it seems important to standardize the room light level at the time of AA measurements. We take that situation into account and all measurements were done at a constant room lightning level. On the other hand, Tonoref III can measure pupil diameter at the time of the AA measurements. This precise control of pupil diameter allows more reliable measurements compared to the other objective and subjective methods. In our study, AA was higher when the pupil diameter was larger. Another finding in our study is that the pupil diameter decreases with age. Thus, we can say that patients with small pupil diameters have lower AA values.

The effects of LASIK on AA have been investigated previously. Liu et al. (23) stated that LASIK treatment did not have a significant effect on AA. Another study by Prakash et al. (24) reported that the AC/A value after LASIK was stabilized between three months and nine months and it was a variable the first three months after LASIK. In our study, when we compared the patients who had been LASIK for myopia at least nine months ago with non-LASIK emmetropic patients in the same age group, we found the AA value was significantly higher in the LASIK group. Since our patient count is low, non-dominant dominant eye distinction like in the first study and myopia degree was not known during LASIK. Our findings should be evaluated with further studies.

Our study has some limitations. We did not evaluate our results with other autorefractometers. Subjective accommodation tests were used only in patients without presbyopia. Another limitation was that accommodation measurements of patients below the age of 25 were not evaluated. Due to the high number of participants, we think that generalization can be made with the result of our study, which is the strongest part of our study.

\section{Conclusion}

Subjective accommodation methods measure the closest distance the patient can see clearly, while objective methods evaluate the actual increase in the eye's refractive power.

The depth of field of the eye may cause overestimated measurement of the AA value in subjective tests. Precise and reproducible objective measurements in AA will help us better understand the accommodation process and guide us in finding new ways to treat presbyopia. AA values measured with the Tonoref III device seem to be suitable for objective measurement of accommodation in presbyopic or non-presbyopic individuals.

\section{Disclosures}

Ethics Committee Approval: The Ethics Committee of TOBB ETU Clinical Research provided the ethics committee approval for this study (24/07/2019 KAEK-1 I8/049).

Peer-review: Externally peer-reviewed.

Conflict of Interest: None declared.

Authorship Contributions: Involved in design and conduct of the study (KO, HK); preparation and review of the study (KO, HK); data collection (KO, HK); and statistical analysis (KO, HK).

\section{References}

I. Win-Hall DM, Glasser A. Objective accommodation measurements in prepresbyopic eyes using an autorefractor and an aberrometer. J Cataract Refract Surg 2008;34:774-84. [CrossRef]

2. Croft MA, Glasser A, Kaufman PL. Accommodation and presbyopia. Int Ophthalmol Clin 200 I;4I:33-46. [CrossRef]

3. Charman WN. The eye in focus: Accommodation and presbyopia. Clin Exp Optom 2008;91:207-25.

4. Anderson HA, Stuebing KK. Subjective versus objective accommodative amplitude: Preschool to presbyopia. Optom Vis Sci 20|4;91:1290-30|. [CrossRef]

5. Wolffsohn JS, Hunt OA, Naroo S, Gilmartin B, Shah S, Cunliffe IA, et al. Objective accommodative amplitude and dynamics with the ICU accommodative intraocular lens. Invest Ophthalmol Vis Sci 2006;47:1230-5. [CrossRef]

6. Ben-Nun J, Alió JL. Feasibility and development of a high-power real accommodating intraocular lens. J Cataract Refract Surg 
2005;3 I:|802-8. [CrossRef]

7. Küchle M, Seitz B, Langenbucher A, Gusek-Schneider GC, Martus P, Nguyen NX; Erlangen Accommodative Intraocular Lens Study Group. Comparison of 6-month results of implantation of the ICU accommodative intraocular lens with conventional intraocular lenses. Ophthalmology 2004; I I I:3 I8-24. [CrossRef]

8. Chen Y, Zhang C, Ding C, Tao C, Bao J, Zheng J, et al. Repeatability of two subjective accommodative amplitude measurements and agreement with an objective method. Clin Exp Optom 2019;102:412-7. [CrossRef]

9. Momeni-Moghaddam H, Wolffsohn JS, Azimi A, BabaeiMalekkolaei E. Effect of target distance on accommodative amplitude measured using the minus lens technique. Clin Exp Optom 2014;97:62-5. [CrossRef]

I0. Momeni-Moghaddam H, Kundart J, Askarizadeh F. Comparing measurement techniques of accommodative amplitudes. Indian J Ophthalmol 2014;62:683-7. [CrossRef]

II. Ovenseri-Ogbomo GO, Kudjawu EP, Kio FE, Abu EK. Investigation of amplitude of accommodation among Ghanaian school children. Clin Exp Optom 2012;95:187-91. [CrossRef]

12. Win-Hall DM, Ostrin LA, Kasthurirangan S, Glasser A. Objective Accommodation Measurement with the Grand Seiko and Hartinger. Optom Vis Sci 2007;84:879-87. [CrossRef]

13. Win-Hall DM, Glasser A. Objective accommodation measurements in pseudophakic subjects using an autorefractor and an aberrometer. J Cataract Refract Surg 2009;35:282-90. [CrossRef]

14. León A, Estrada JM, Rosenfield M. Age and the amplitude of accommodation measured using dynamic retinoscopy. Ophthalmic Physiol Opt 2016;36:5-12. [CrossRef]

15. Win-Hall DM, Houser J, Glasser A. Static and Dynamic Accom- modation Measured. Optom Vis Sci 2010;87:873-82. [CrossRef]

16. Desmond T, Arthur P, Watt K. Comparison of central corneal thickness measurements by ultrasound pachymetry and 2 new devices, Tonoref III and RS-3000. Int Ophthalmol 2019;39:9I723. [CrossRef]

17. Singman E, Matta N, Tian J, Silbert D. Association between accommodative amplitudes and amblyopia. Strabismus 2013;21:137-9. [CrossRef]

18. López-Alcón D, Marín-Franch I, Fernández-Sánchez V, LópezGil N. Optical factors influencing the amplitude of accommodation. Vision Res 2017;141:16-22. [CrossRef]

19. McBrien NA, Millodot M. Amplitude of accommodation and refractive error. Invest Ophthalmol Vis Sci 1986;27:I 187-90.

20. Abraham LM, Kuriakose T, Sivanandam V, Venkatesan N, Thomas R, Muliyil J. Amplitude of Accommodation and its Relation to Refractive Errors. Indian J Ophthalmol 2005;53: 105-8.

2I. Schaeffel F, Wilhelm H, Zrenner E. Interindividual variability in the dynamics of natural accommodation in humans: relation to age and refractive errors. J Physiol 1993;46I:30I-20. [CrossRef]

22. Lara F, Bernal-Molina P, Fernández-Sánchez V, López-Gil N. Changes in the objective amplitude of accommodation with pupil size. Optom Vis Sci 2014;91:1215-20. [CrossRef]

23. Liu L, Yuan J, Li J, Li X, Wang Y. Effect of laser in situ keratomileusis on accommodation. J Huazhong Univ Sci Technolog Med Sci 2008;28:596-8. [CrossRef]

24. Prakash G, Choudhary V, Sharma N, Titiyal JS. Change in the accommodative convergence per unit of accommodation ratio after bilateral laser in situ keratomileusis for myopia in orthotropic patients. Prospective evaluation. J Cataract Refract Surg 2007;33:2054-6. [CrossRef] 